\title{
PROPERTIES OF TOXINS OF VERTICILLIUM DAHLIAE, THE CAUSATIVE AGENT OF COTTON WILT DISEASE
}

\author{
N.A. KRASSILNIKOV, C.M. KHODJIBAYEVA AND T.G. MIRCHINK \\ Department Biology of Soil, University of Moscow, Moscow, \\ Leninsky Gory U.S.S.R.
}

(Received May 11, 1968)

1. Determination of the toxicity of the culture liquid of Verticillium dahliae showed that it contains an active toxin which causes wilting of cotton plant shoots. Thus the fungus produces a toxin which it excretes into the external medium.

2. By testing a large number of strains of microorganisms from various taxonomic groups, a test microbe was found which is sensitive to the toxin of Verticillium dahliae.

3. Such a sensitive test-microbe made it possible to use chromatography with a bioassay method to select the best solvent for extraction of the toxin.

4. The toxin was obtained in concentrated form by extraction with amyl acetate.

5. A study of the physico-chemical properties of the toxin showed it to be acidic in nature, and that it has two sharp absorption maxima in the UV region at 245 and $275 \mathrm{~m} \mu$, and is stable at high temperatures and over a wide range of $\mathrm{pH}$ values.

6. The toxin of Verticillium dahliae is composed of two fractions, differing in physico-chemical and biological properties.

7. The toxin of Verticillium dahliae does not appear to be highly specific, and, besides its marked inhibitory action on cotton, causes wilting of leguminous plants, during which time cereals are not sensitive to the toxin in the concentrations tested.

8. The rate of wilting under the influence of the toxin depends on the age and variety of cotton plant. Adult plants wilt more quickly than young plants. The cotton plant variety 108-F (resistant to wilting) is less sensitive to the toxin than variety 2034 , which is non-resistant to wilting. The toxin causes browning of the vascular bundles and the surrounding woody parts of the stalk, and the leaves loose their turgidity.

9. The tetraene antibiotics, $18-45$ and $18-80$, produced by actinomycetes, have an effect on the toxin produced by Verticillium dahliae. They interact with the fungal toxin and neutralise its action.

10. Treatment of seeds with antibiotics or cultivation in soils which have been enriched with the antibiotic-producing actinomyces produces an immunity in the cotton plants which is passed on to the following generations. 
At present there is still no general agreement concerning the reasons for wilting of plants which have become infected by fungi. Among the earliest conceptions of this phenomenon, mechanical blockage of the vascular system by fungal hyphae or accumulation of toxic compounds formed by fungi in diseased plants was suggested as a reason for plant wilting (1-3). Recently, several authors have attributed such a role to glucosine (4). An important opinion is that plant wilting is due to active enzymes of the parasitic fungi which cause direct injury of tissues and disruption of cells $(5,6)$. More generally accepted at present however is the theory of intoxication, which states that plant disease is due to toxins excreted by the phytopathogen fungi into the external medium. A more detailed account of this phenomenon is to be found in the monograph by GAUMANN (4).

Many fungi are known to form different toxic products, some of which do not possess any kind of selective activity towards plant species (toxins with non-specific activity) and others which, on the contrary, are highly specific in their activity. Substances of a carbohydrate or protein nature, organic acids, and other compounds belong to the first group. Licomarazmin, a compound toxic to tomato plant, is an example of a specific toxin, being produced only by one fungus, Fusarium lycopersici (7). Victorin, isolated from the cultures of Helminthosporum victoriae, causes disease only in certain kinds of oats $(8)$. A toxic substance towards sorghum has been obtained from Periconia cireinata (9). Such toxins are essentially the cause of plant diseases. Their action on a given plant can reproduce all symptoms of a disease which would normally develop during a natural attack on the plant by the pathogenic fungus. They are agent of the disease.

However, the mode of action of such a toxin as fusaric acid (a compound produced by several species of the genus Fusarium, certain members of which are the agents of plant wilting) is somewhat different. With fusaric acid it is impossible to completely reproduce all symptoms of wilting, which fact suggests that the disease is not entirely due to the action of this acid (10). Also, the cause of cotton wilt disease by Verticillium dahliae is not fully understood. Lately, more and more evidence is accumulating which suggests that the wilting and destruction of cotton by Verticillium dahliae are also due to the toxin production by this fungus in the external medium $(6,11,12)$. However so far there has been no information concerning the chemical nature and properties of these substances.

The elucidation of the chemical nature and biological properties of the toxins is of prime importance in solving the mode of action of Verticillium dahliae in the disease of the cotton plant, and in elaborating effective methods for the prevention of wilting. Thus, there is foundation for work demonstrating the toxin of Verticillium dahliae and studying of its physico-chemical properties. 


\section{METHODS}

A culture of Verticillium dahliae, obtained from the Botanical Institute, Academy Sciences of U.S.S.R., was used in experiments concerned with the isolation of the toxin.

Toxicity of the cultural liquid of the fungus was determined on cuttings of the cotton plant, by submersion of the cuttings into the culture liquid. The culture liquid was obtained by growing Verticillium dahliae in malt extract medium for 5 days in $100 \mathrm{ml}$ of medium contained in $700-\mathrm{ml}$ flasks, which were shaken during the incubation period. After 5 days, the culture liquid was filtered and the cotton cuttings were submerged into it. In the control, cotton shoots were placed in tap water which had been acidified to the same $\mathrm{pH}$ as that of the cultural liquid $(\mathrm{pH} 5.0)$. The shoots were maintained in the culture liquid for 3 days during which time the rate of wilting was observed. Toxicity of the culture liquid was estimated from the rate of wilting of the shoots.

A paper chromatography method, used previously for antibiotics (13), was employed for selection of the best solvent for extraction of the toxin. The method of ascending chromatography was used, and included 14 solvent systems. To demonstrate the presence of the toxin on the chromatograms, a bioassay procedure with a test microbe was used. The concentrate obtained was studied with the help of electrophoresis and spectrophotometry. The UV spectrum of the concentrate was measured in a model SF-4 spectrophoto-

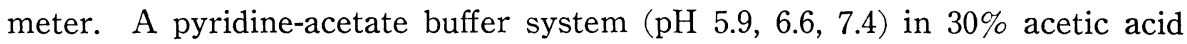
$(\mathrm{pH} 2)$ was used for the electrophoresis.

\section{RESULTS}

After $9-10 \mathrm{hr}$ there were noticeable signs of wilting of the cotton plants which had been submerged into filtered culture liquid of Verticillium dahliae. The wilting became more and more acute as time passes. The leaves shrank, lost their turgidity, and wilted. After the second day the shoots were completely wilted, whereas control plants remained without change during this time. This fact suggested that there may be factors in the culture liquid which caused wilting. Work was then carried out with the aim of extracting the toxin which causes wilting of the cotton shoots.

The culture liquid was chromatographed and the best solvent was selected for subsequent extraction of the toxin. As the chromatograms were tested by a bioassay method with a test microbe, an organism had to be selected which was sensitive to the toxin of Verticillium dahliae and 125 strains of microorganisms from different taxonomic groups were tested; 30 species of bacteria and mycobacteria, 14 species of fungi, 33 species of actinomycetes, and one species of algae, Chlorella. From all of these microorganisms tested, only one culture, Micromonospora vulgaris strain 1005, proved to be sensitive to the toxin secreted into the culture liquid by Verticillium dahliae. The 
results of chromatography of the culture liquid of Verticillium dahliae with the use of the test microbe Micromonospora vulgaris are shown in Table 1.

Table 1. $R_{f}$ value of toxins in the different system of solvents.

\begin{tabular}{l|l}
\hline \multicolumn{1}{c|}{ Solvents } & \multicolumn{1}{c}{$R_{f}$} \\
\hline Acetone $80 \%$ & $0 ; 0.9$ \\
Methanol-isoamy' acetate-water $(24: 14: 1)$ & 0.95 \\
Butanol-hexane-acetone-water $(20: 9 \cdot 10: 1)$ & 0.9 \\
Acetone-benzene-water $(30: 9: 1)$ & $0 ; 0.6 ; 0.8$ \\
Distilled water & $0 ; 0.8$ \\
Amyl acetate & 0.3 \\
Butanol-methanol-water $(4: 1:\{)$ & 0.9 \\
CCL 4 & 0 \\
Petroleum ether & 0 \\
Benzene, water-saturated & 0.1 \\
Ethyl acetate, water-saturated & $0.5 ; 0.95$ \\
Chloroform, water-saturated & 0.95 \\
Butanol, water-saturated & $0.5 ; 0.95$ \\
Isopropanol-chloroform-benzene-water $(30: 4: 4: 2)$ & 0 \\
\hline
\end{tabular}

The data obtained from chromatography of the toxin showed that this substance is very soluble in $80 \%$ acetone, ethyl acetate, chloroform, butanol, distilled water, and amyl acetate; only very slightly soluble in benzene, and insoluble in carbon tetrachloride and petroleum ether. In such systems as acetone-benzene-water and ethyl acetate-butanol, toxin is separated into two fractions. On the basis of the chromatography data, amyl acetate $\left(R_{f}=0.3\right)$ was selected for extraction of the toxin.

Before extraction, the filtered culture liquid was acidified with $2 \mathrm{~N} \mathrm{HCl}$ to $\mathrm{pH}$ 3.0. Without acidification, the toxin could not be extracted. One volume of amyl acetate was added to 2 volumes of culture liquid and the mixture was carefully shaken for $1 \mathrm{hr}$ and the solvent was separated from the culture liquid. This was followed by a second extraction in which 1 volume of amyl acetate was added to 3 volumes of previously extracted culture liquid. The combined extract was then centrifuged at $1,500 \mathrm{rpm}$ for $15 \mathrm{~min}$ and then filtered through filter paper to separate the emulsion which formed during the extraction procedure. The emulsion obtained in this manner, like the concentrate, was found to be active and was used for further investigations. It was soluble in water, ethanol, amyl acetate, and other solvents.

The concentrate was evaporated under vacuum at $40^{\circ}$ to $1 / 100$ of its original volume and tested for toxic activity by its action on the cotton plant and on the test microbe. 
To test the activity of the toxin on the plant, a series of dilutions of the concentrate were prepared into which cotton shoots were introduced. To determine the activity against the test microbe, various dilutions of the concentrate were pipetted onto chromatography paper, which were then applied to an agar surface sown with Micromonospora vulgaris.

Results showed that cotton shoots were more sensitive than Micromonospora to the toxin. The concentrate was toxic to cotton shoots in a dilution of $1: 1,000$, and to the microorganism in a dilution of $1: 20$.

Certain physicochemical properties of the concentrate were examined. The toxin proved to be stable at high temperatures; boiling for $20 \mathrm{~min}$ did not reduce the activity of this compound. Changes in $\mathrm{pH}$ of the medium from 2 to 10 did not cause any fundamental change in its properties. With the help of electrophoresis it was established that the substance is acidic in nature.

The determination of ultraviolet spectrum of the concentrate showed the presence of two sharp absorption maxima in the region of 245 and $275 \mathrm{~m} \mu$ (Fig. 1). As was mentioned above, in certain solvent systems (acetonebenzene-water, ethyl acetate, and butanol) there is a separation of the substance into two fractions, and these two fractions were eluted with amyl acetate.

First, the toxin was chromatographed with the solvent system of acetone: benzene: water $(30: 9: 1)$. Each of these fractions was subjected to electrophoresis from which it was established that both compounds are acidic in character. On measuring the UV spectrum of the fractions, one of the latter,

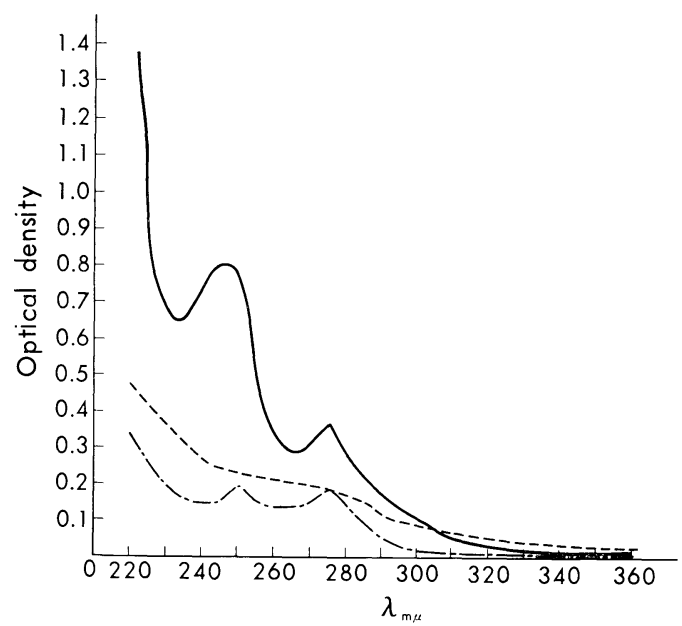

Fig. 1. UV absorption spectrum of the toxin (in alcohol).

$\begin{array}{ll} & \text { common concentration } \\ \ldots-\ldots & \text { fraction I } \\ \ldots-.- & \text { fraction II }\end{array}$


with an $R_{f}$ of 0.6 , was found to have absorption maxima in the region of 250 and $275 \mathrm{~m} \%$ (Fig. 1). The other fraction, which had $R_{f}$ of 0.8 , did not have well defined absorption peaks.

The two compounds do not essentially differ from each other in solubility, and are stable at high temperatures and over a wide range of $\mathrm{pH}$ (from 2 to 10).

\section{Action of Toxins on Plants}

Effect of the two separated fractions of the toxin concentrate on the plant was then studied, with dilutions of the toxin concentrate in $1: 10,000$, $1: 1,000$, and $1: 100$.

It was observed that the rate of wilting depends on the age and kind of the cotton plant. Mature plants begin to wilt significantly earlier than young plants. Cotton plant Type 108-F (resistant to wilting) is less sensitive to the action of the toxin than Type 2034, a strain non-resistant to the wilt disease. Dilution 1:10,000 was almost without effect on cotton Type 108-F. Dilution $1: 1,000$ caused wilting within $9-10 \mathrm{hr}$. With dilution $1: 100$, which has a very marked effect on the plant, wilting developed within 5-6 hr. Cotton Type 2034 begins to wilt significantly earlier; i.e. within $18-19 \mathrm{hr}$ at $1: 10,000$, within $5-6 \mathrm{hr}$ at $1: 1,000$, and within $2-3 \mathrm{hr}$ at $1: 100$.

When the plants were treated with the toxin from Verticillium dahliae, browning of the vascular bundles and the surrounding parts of the wood of

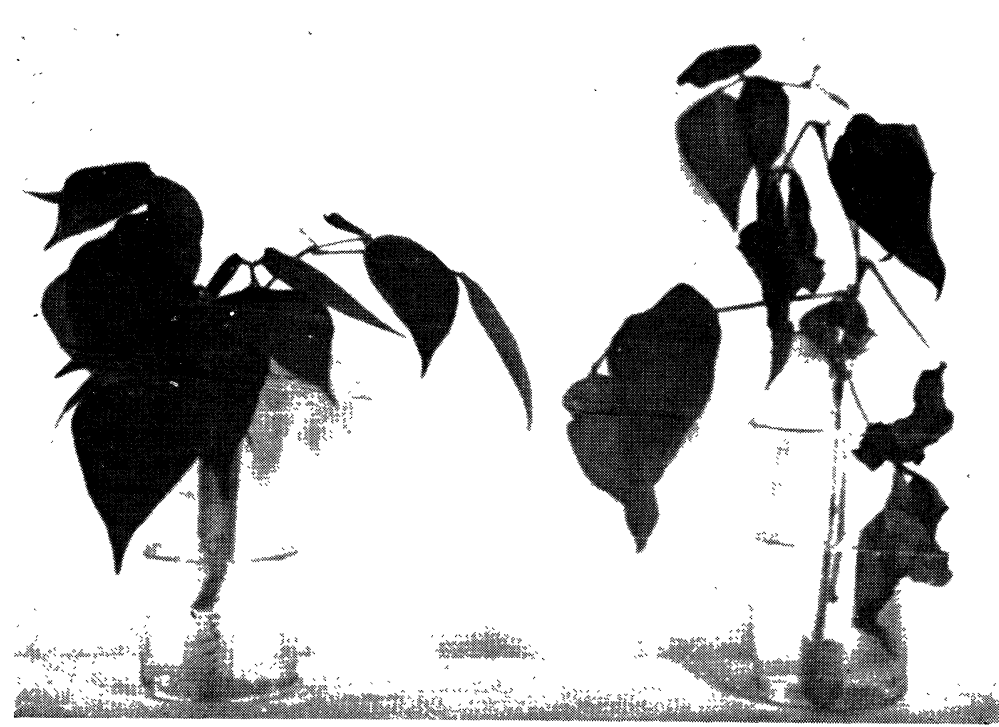

Fig. 2. Effect of the toxin on cotton shoots.

b
a) control
b) solution of the toxin water 
the stalk, and loss of turgidity of the leaves were observed. On testing the action of the separate fractions of the toxin, it was found that both substances caused changes in the plant, typical of the cotton wilt disease. Characteristic symptoms of the disease developed and the plants soon perished. The action of the toxin was tested on a series of other plants. When grown plants of cotton were treated with the toxin, it was absorbed by roots and entered in the stems and leaves, the highest concentration being in the roots, and lower in the stems and leaves (Fig. 3). In addition to the separation and study of the physicochemical properties of the toxin, antagonistic properties of actinomyces strains were tested, with the aim of finding an antibiotic which would be active against this fungus that causes the wilt disease. A large number of actinomycetes antagonistic to Verticillium dahliae were found. These antibiotics are polyene derivates. Two tetraene antibiotics, obtained from strains $18-45$ and $18-80$ and belonging to the group of "Albus" actinomycetes, were chosen for a more detailed study as they were highly active in relation to the prevention of cotton wilt disease (14). A determination was made of the ability of these antibiotics to inactivate the toxin formed by Verticillium

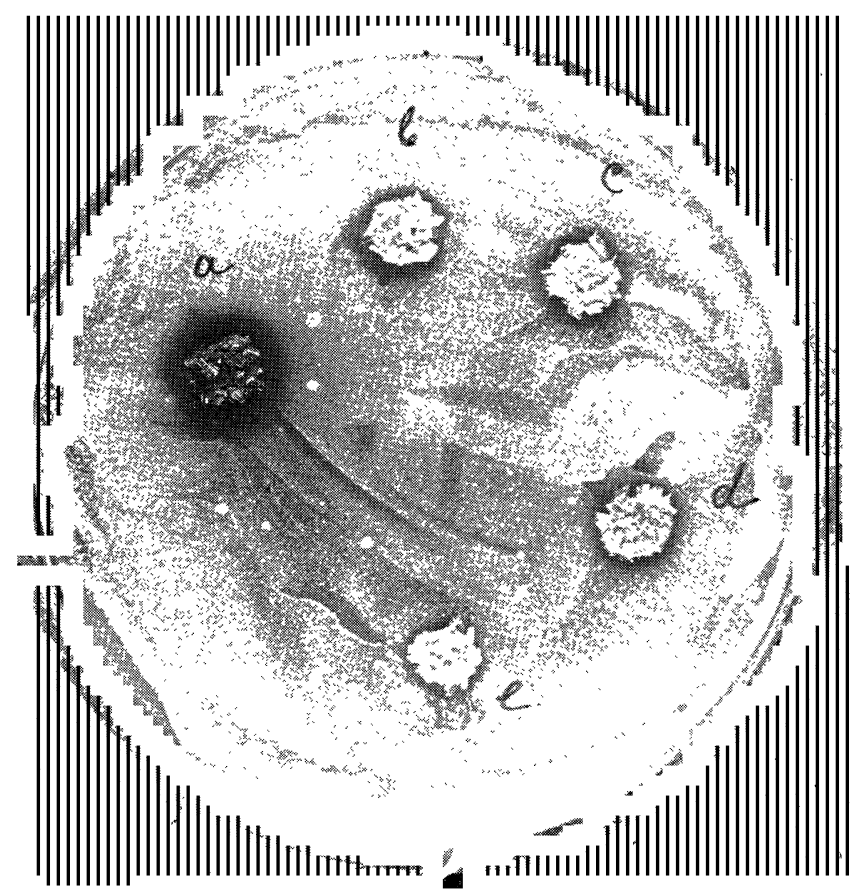

$\mathrm{Fi} .2$ Antibiotic activity of the plant treated with antibiotic 18-45 stem of the plant on a plate of Micromonospora vulgaris.
a) roots
b, c) stem-lower part
d) stem-higher part
e) leaf 
dahliae. One milliliter of the toxin was added to $1 \mathrm{ml}$ of the culture liquid of Actinomycete (Strain 18-80 or 18-45), and this mixture was kept for $1 \mathrm{hr}$ before being tested for toxicity against the cotton plant and the thermophilic actinomycetes, Micromonospora vulgaris. Two controls were set up, the first with cotton shoots submerged in a solution of pure toxin, and the second with the shoots in tap water. For the test on Micromonospora vulgaris, such a mixture was pipetted into a small cylinder on agar medium seeded with this organism. For the control, pure toxin was used without the antibiotic.

These experiments showed that the addition of crude antibiotic or its concentrate to the toxin completely eliminated the toxic action of the test solution. Plant treated with this mixture did not show any signs of wilting (Fig. 4). During the same period cotton shoots submerged in a solution of pure toxin showed definite signs of wilting, characteristic of the disease, and plants submerged into tap water remained healthy.

These antibiotics had a similar action in experiments using the test microbe. If a mixture of the toxin and antibiotic was placed on an agar medium seeded with Micromonospora vulgaris, no sterile zones of growth inhibition were formed during the time when pure toxin produced noticeable zones of growth inhibition.

Thus the antibiotics 18-45 and 18-80 not only inhibit the development of the causative agent of wilt, but also neutralise the toxin formed by this fungus.

Thus the cultures $18-45$ and $18-80$ produce a substance with double action; antibiotic and antitoxic. It is possible that there was a mixture of two metabolites in these preparations, as the latter was not particularly pure. It should be mentioned that the phenomenon of neutralisation of toxins of fungal and bacterial origins was observed by KRASSILNIKOV (15) with a series of antibiotics. Apart from their antibiotic and antitoxic properties, these

a

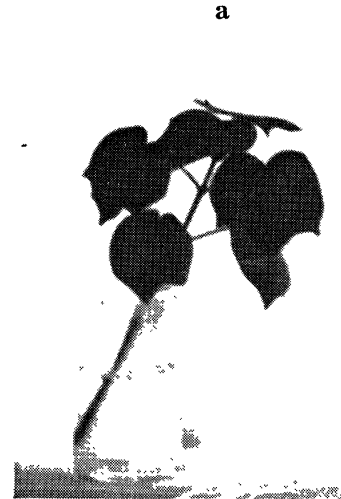

b

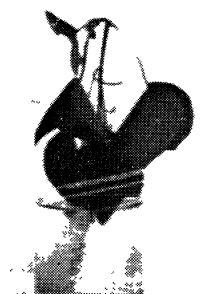

c

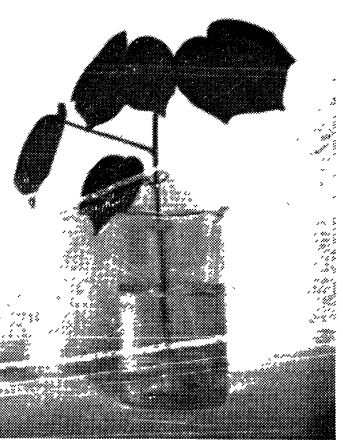

Fig. 4. Inactivation of the toxin by the antibiotic-antitoxin.
a) control
b) effect of the toxin
c) effect of toxin and antitoxin 
antibiotics influence the immunity of the plant.

Cotton plants were cultivated in soil which had been previously enriched with actinomycetes composts, after which a determination of the fungicidal power of the cell sap of various organs of the mature plant was made. Crushed preparations of various organs of the plant were added to the centre of an agar surface, seeded with Verticillium dahliae. These experiments demonstrated that during the period when the sap of the control plants showed no fungicidal properties, plants grown in soil enriched with actinomycetes acquired these properties. Moreover, it was observed that plants grown from cotton seeds treated with the antibiotic were less susceptible to fungal infection.

Seeds of a cotton plant, which had previously been cultivated in a soil containing actinomycetes fertiliser, shows a normal growth. As a control, seeds of plants grown on ordinary soil without actinomycetes fertiliser were sown. After the plants developed to the 4-6 leaflet stage, the cotton plants were artificially contaminated with Verticillium dahliae. All the plants in the control acquired the wilt disease, while, when added with actinomycetes, only thirty-four per cent of the test plants was diseased with wilt. Thus, the use of these antibiotics enables the plants to acquire immunity in the following generation.

\section{REFERENCES}

1) G. Schiccora, Arb. der Kaiserl. Biol. Aust. fur Land und Forstwirtsch., 5, 157 (1906).

2) W.F. Bewley, Ann. Appl. Biology, 11, 116 (1922).

3) B.A. Rudolph, Hilgardia Journ. of Agr. Sci., 5, (9), 201 (1931).

4) E. Gaumann, Publishing House of Foreign Literature, Moscow. (1954).

5) S.S. Gothoskar, R.P. Scheffer, T.C. Walker and O. Stahmayn, Phytopathology, 43. 535 (1953).

6) M. Kamal and R. Wood, Ann. Appl. Biol., 44, N2, 322 (1956).

7) N. Clauson-KaAs, P. Platter and E. Gaumann, Ber. Schweiz. Bot. Ges., 54, 523 (1944).

8) H.E. Wheeler and H.H. Luke, Phytopathol., 44, 334 (1954).

9) R.P. ShefFer and R.B. PRINGLE, Nature, 191, N 4791, 912 (1961).

10) H.E. Wheeler, Ann. Rev. of Microbiology, p. 17 (1963).

11) P.W. Talboys, Trans. Brit. Mycol. Soc., 40, 415 (1957).

12) R.R. Sheffer, S.S. Gothoskar, C.F. Pierson and R.P. Collins, Phytopathology, 46, 83 (1956).

13) N.O. Blinov and A.S. KhoKhlov, Antibiotiki, 2, 183 (1962).

14) S.M. Khodjibaeva, Microbiologiya, 33, No. 4, 661 (1964).

15) N.A. Krassilnikov, Academy of Sciences, Moscow (1958). 